\title{
From contamination to infective endocarditis-a population-based retrospective study of Corynebacterium isolated from blood cultures
}

\author{
Magnus Rasmussen ${ }^{1,2,3}$ (D) Anna Wramneby Mohlin ${ }^{1} \cdot$ Bo Nilson $^{4,5}$
}

Received: 27 June 2019 / Accepted: 30 August 2019 / Published online: 4 September 2019

(C) The Author(s) 2019

\begin{abstract}
Corynebacterium is a genus that can contaminate blood cultures and also cause severe infections like infective endocarditis (IE). Our purpose was to investigate microbiological and clinical features associated with contamination and true infection. A retrospective population-based study of Corynebacterium bacteremia 2012-2017 in southern Sweden was performed. Corynebacterium isolates were species determined using a matrix-assisted laser desorption/ionization-time-of-flight mass spectrometry (MALDI-TOF MS). Patient were, from the medical records, classified as having true infection or contamination caused by Corynebacterium through a scheme considering both bacteriological and clinical features and the groups were compared. Three hundred thirty-nine episodes of bacteremia with Corynebacterium were identified in 335 patients of which $30(8.8 \%)$ episodes were classified as true infection. Thirteen patients with true bacteremia had only one positive blood culture. Infections were typically community acquired and affected mostly older males with comorbidities. The focus of infection was most often unknown, and in-hospital mortality was around $10 \%$ in both the groups with true infection and contamination. Corynebacterium jeikeium and Corynebacterium striatum were significantly overrepresented in the group with true infection, whereas Corynebacterium afermentans was significantly more common in the contamination group. Eight episodes of IE were identified, all of which in patients with heart valve prosthesis. Six of the IE cases affected the aortic valve and six of seven patients were male. The species of Corynebacterium in blood cultures can help to determine if a finding represent true infection or contamination. The finding of a single blood culture with Corynebacterium does not exclude true infection such as IE.
\end{abstract}

Keywords Corynebacterium $\cdot$ Bacteremia $\cdot$ Infective endocarditis $\cdot$ Contamination $\cdot$ Blood culture

\section{Introduction}

Corynebacterium is a genus of Gram-positive rods comprising more than 100 species of which a large proportion has

Magnus Rasmussen

magnus.rasmussen@med.lu.se

1 Section for Infection Medicine, Department of Sciences Lund, Lund University, Lund, Sweden

2 Division for Infectious Diseases, Skåne University Hospital, Lund, Sweden

3 Division of Infection Medicine Diseases, Department of Clinical Sciences Lund, Lund University, BMC B14, SE-223 63 Lund, Sweden

4 Department of Clinical Microbiology, Labmedicin, Region Skåne, Lund, Sweden

5 Division of Medical Microbiology, Department of Laboratory Medicine Lund, Lund University, Lund, Sweden been associated to human infections [1]. Corynebacterium diphtheriae is the most well-known of these species, but over the last decades, more attention has been drawn to nondiphtheriae Corynebacterium species as human opportunistic pathogens [1].

When Corynebacterium is isolated from blood cultures, the finding is often dismissed as a contamination from normal skin flora rather than recognized as a pathogen causing true infection. Studies investigating different cohorts have indicated that between 44 and $71 \%$ of patients with Corynebacterium bacteremia have true infection [2-4]. Some studies have only used a bacteriological criterion ( $\geq 2$ positive blood cultures) $[2,3]$ whereas other studies also considered clinical features and acknowledge that intravascular devices are risk factors for true Corynebacterium infection [4-6]. The lack of a uniform definition of contamination and infection hampers the possibility to draw conclusions about the incidence of true Corynebacterium infections. Moreover, available studies 
describe limited number of cases and have a retrospective design $[2-5,7]$.

Corynebacterium can cause infective endocarditis (IE) and is a rare etiology of such infections [8]. Knowledge on Corynebacterium IE comes mainly from case reports or systematic reviews of the literature. From a large systematic review it was concluded that Corynebacterium IE mainly affects the left side of the heart (95\% of cases) and that men constituted $72 \%$ of described cases [9]. In this case review, $19 \%$ of cases represented valve prosthesis IE and the mortality rate was high (40\%) [9]. Interestingly, a difference in clinical presentation between different species of corynebacterial was noted [9].

Species determination of corynebacteria has been challenging [1] but the introduction of matrix-assisted laser desorption/ ionization-time-of-flight mass spectrometry (MALDI-TOF MS) has provided a practical tool for this purpose $[10,11]$. It has been shown that species determination of Corynebacterium can be performed with sufficient precision using improved databases and lower cutoff scores in the analysis of spectra [11]. Several studies have investigated the features of infections with specific Corynebacterium species. Of non-diphtheriae corynebacteria, Corynebacterium striatum and Corynebacterium jeikeium have often been reported as pathogens $[3,5,7,9,12]$. However, at present, there is insufficient data on how species determination of corynebacteria can help to determine the risk for true infection or even IE in patients with Corynebacterium bacteremia.

The present study aims to determine risk factors for true Corynebacterium infection, including IE, in a large retrospective population-based cohort of patients with positive blood cultures.

\section{Methods}

\section{Microbiology and species determination}

Data on patients with blood cultures positive for Corynebacterium were collected from the registry of the Laboratory for Clinical Microbiology in the county of Skåne, Sweden, for the years 2012 to 2017. A blood culture was regarded to be positive irrespective of if one or two bottles yielded growth. The laboratory is the only one in Skåne, a province with a population of $1,329,000$ inhabitants (January 1, 2017, data from Statistics Sweden (available at https://www.scb.se), and all cultures from this province (including nine hospitals and all primary care facilities) are handled by our laboratory. From 2012 through late 2014, the BacT/Alert blood culture system (bioMérieux, Marcy l'Etoile, France) was used and was replaced by the BACTEC FX blood culture system (Becton Dickinson, Franklin Lakes, USA) in December 2014. The laboratory receives approximately
75,000 blood culture bottles/year of which roughly $12 \%$ grow bacteria. The isolates had been identified by MALDITOF MS and the majority of them were identified at genus level only. For the study, stored isolates were re-cultured on blood agar plates under aerobic conditions, and when sufficient growth was observed (at between 24 and $120 \mathrm{~h}$ ), samples were analyzed by the direct colony method using MALDITOF MS (version Microflex MALDI-TOF MS, software FlexControl 3.4 and MALDI Biotyper (MBT) Compass 4.1, with MBT Compass Library, DB-7854 MSP (Bruker, Bremen, Germany). In cases where a low score was achieved, we also used a standard ethanol-formic acid extraction method described by the instrument manufacturer. The cutoff of 1.7 for species determination as suggested by Alatoom et al. [11] and also others [13] was employed. Resistance was determined according to EUCAST protocols, and breakpoints were defined according to EUCAST guidelines (available at EUCAST.org).

\section{Patients and definitions}

Medical records were studied retrospectively. Records inaccessible and patients under the age of 18 were excluded from further analysis. The regional ethics committee of Lund University approved of the study (2017/1002). An episode was defined as a clinical situation where Corynebacterium was isolated from blood and the episode was concluded after 2 weeks of effective antibiotic treatment. Thus, if Corynebacterium was isolated from the same patient within 14 days, this was regarded as persistent bacteremia whereas a positive culture after 14 days was regarded as a new episode. Data collected included age, gender, the use of immunosuppressive treatment, underlying medical conditions according to Charlson [14], and criteria for IE according to Duke [15]. Nosocomial infections were defined as an infection evident at $48 \mathrm{~h}$ or more past hospital admission. Focus of infection was defined by fulfillment of the following three criteria (i) the isolation of the relevant bacterium from the site of infection, (ii) signs and symptoms of focal infection, and iii) radiological signs of focal infection [16]. In-hospital mortality was recorded.

We modified the definitions for discrimination of true infection from contamination described previously by Finkelstein and co-workers [6] and presented our definition in Table 1. For episodes with two or more blood cultures with growth of Corynebacterium, signs of infection (one of the following: temperature $\geq 38{ }^{\circ} \mathrm{C}$, systolic blood pressure < $100 \mathrm{mmHg}$, presence of chills or leukocytosis $\left(>12 \times 10^{9} /\right.$ L)) were needed for the episode to be classified as true infection (criterion 1). True infection was rejected if another more likely cause of the infection, such as a positive blood culture with more pathogenic bacteria (criterion 2) or a focal infection where another bacterial species was deemed to be a more 
Table 1 Definitions of true infection depending on the number of positive cultures

Two or more positive blood cultures

Criterion 1 Infection confirmed by temperature $>=38$ OR hypotension (systolic blood pressure $<100 \mathrm{mmHg}$ ) OR chills OR leukocytosis $\left(>12 \times 10^{9} / \mathrm{L}\right)$

Criterion 2 No other more likely pathogen in blood culture explains confirmed infection

Criteria 3 No other focal infection where other bacteria are more likely explains symptoms of patient, where focal infection is defined by two of the following $(\mathrm{a}-\mathrm{c})$ :

a. Isolation of pathogens other than Corynebacterium at site of infection

b. Typical signs or symptoms of focal infection

c. Imaging results compatible with focal infection

Additional criteria in cases with one positive blood culture

Criteria 4 Foreign intravascular device ${ }^{\mathrm{a}}$ present $>48 \mathrm{~h}$ prior to blood sample OR Corynebacterium isolated at site of infection

${ }^{a}$ Central venous catheters, port-á-cath, implantable cardioverters, pacemakers, prosthetic heart valves, intravascular grafts, and picc-lines

likely cause (criterion 3). If another bacterium was isolated from the site of infection or if the focal infection was pneumonia, soft tissue infection, or urinary tract infection, this excluded true Corynebacterium infection. If the Corynebacterium was isolated from the site of infection or if the focal infection was IE, spondylitis, and arthritis with no findings of bacteria from the focal infection, Corynebacterium was regarded as the most likely etiology. To classify episodes with only one blood culture with Corynebacterium as true infection, the episode had to fulfill criteria $1-3$, and in addition, an intravascular device had to be at place for more than $48 \mathrm{~h}$ or Corynebacterium had to be isolated from the site of infection. Episodes of Corynebacterium bacteremia not fulfilling above criteria were regarded as contamination.

\section{Statistical analysis}

For categorial data, statistical analysis was performed using two-sided $\chi^{2}$ test (Pearson) for multiple comparisons or Fischer's exact test for pairwise comparisons. Continuous variables were investigated for difference using Mann-Whitney $U$ test. A $p$ value of $<0.05$ was regarded as statistically significant.

\section{Results}

\section{Description of the cohort}

Four hundred twelve blood cultures with growth of Corynebacterium were identified in 369 patients with 373 episodes of bacteremia. Ten episodes were excluded because of inaccessible medical records and 24 episodes because of patient age under 18 years. This resulted in a total of 335 patients with 339 episodes in the final analysis. Thirty $(8.8 \%)$ episodes were classified as true infection, whereas $309(91 \%)$ episodes were classified as contamination. Characteristics of the clinical features of episodes are listed in Table 2. Both groups were predominantly elderly males with comorbidities. Corynebacterium was cultured $(n=6)$ or detected by $16 \mathrm{~S}$ rRNA gene PCR and DNA sequencing ( $n=$ 3) from the site of focal infection in nine episodes in which four patients had IE, and one each had spondylodiscitis, joint prosthesis infection, septic arthritis, pneumonia, central-line infection, and urinary tract infection.

\section{Identification and characterization of isolates}

Two hundred fifty-eight of 339 (76\%) isolates could be classified to the species level; remaining isolates had either not been saved or could not be recultured from stocks. The most abundant species identified in the episodes representing true infections and contaminations are given in Table 3. Corynebacterium afermentans was the most commonly isolated species in episodes representing contamination, whereas C. striatum was the most common species in cases of true infections. Despite its low frequency in the microbiological data set, four of seven episodes with C. jeikeium were categorized as true infection. The difference in species distribution between the groups was statistically significant $(p<0.0001$ in chi-square test for difference).

Of isolates from true infections, the majority were resistant to penicillin and clindamycin (25 and 24 of 30 tested isolates, respectively), whereas a majority of isolates were sensitive to fluoroquinolones and rifampicin (13 of 24 and 18 of 21 isolates, respectively). All isolates tested were sensitive to vancomycin $(n=30$, median MIC 0.5) and linezolid $(n=22)$.

\section{IE caused by Corynebacterium}

Eight episodes of bacteremia were regarded and treated as IE (see Table 4 for clinical descriptions). C. jeikeium and $C$. striatum were responsible for two cases each whereas $C$. propinquum, $C$. amycolatum, and C. pseudodiphtericum were responsible in one patient 
Table 2 Characteristics of patients with true infection and contamination

\begin{tabular}{|c|c|c|c|}
\hline & True infection $(n=30)$ & Contamination $(n=309)$ & $p$ value for difference \\
\hline Age years $\left(\mathrm{IQR}^{1}\right)$ & $74(69-80)$ & $75(63-83)$ & 1 \\
\hline Female gender, $n(\%)$ & $9(30)$ & $129(38)$ & 0.2 \\
\hline \multicolumn{4}{|l|}{ Charlson score } \\
\hline $0-1$ & $7(23)$ & $129(42)$ & \multirow[t]{3}{*}{0.1} \\
\hline $2-3$ & $12(40)$ & $105(34)$ & \\
\hline$\geq 4$ & $11(37)$ & $75(24)$ & \\
\hline Immunosuppressive treatment & $5(17)$ & $31(10)$ & 0.3 \\
\hline Intravascular device & $20(67)$ & $37(12)$ & $<0.0001$ \\
\hline Community site of acquisition & $25(83)$ & $278(90)$ & 0.3 \\
\hline \multicolumn{4}{|l|}{ Focus of infection } \\
\hline Unknown & $19(63)^{2}$ & $165(53)^{3}$ & \multirow[t]{9}{*}{$\mathrm{ND}^{4}$} \\
\hline IE & $5(17)$ & 0 & \\
\hline Bone and joints & $3(10)$ & $3(1.0)$ & \\
\hline Lungs & $1(3.3)^{5}$ & $63(20)$ & \\
\hline Urinary tract & $1(3.3)^{5}$ & $24(7.8)$ & \\
\hline Central-line & $1(3.3)^{5}$ & 0 & \\
\hline Skin/soft tissue & 0 & $28(9.1)$ & \\
\hline Abdominal & 0 & $20(6.5)$ & \\
\hline Other & 0 & $6(1.9)$ & \\
\hline In-hospital mortality & $3(30)$ & $33(11)$ & 1 \\
\hline
\end{tabular}

${ }^{1}$ Inter-quartile range. ${ }^{2}$ Includes three episodes of possible IE. ${ }^{3}$ Unknown focus or no infection. ${ }^{4}$ Not determined due to too few observations in some cells. ${ }^{5}$ Corynebacterium isolated at the site of infection

(of whom one had two episodes) each. In five cases, Duke's criteria for definite IE were fulfilled, and in one patient with possible IE, ${ }^{\text {FDG }}$ PET-CT showed strong uptake in relation to the valve prosthesis. Interestingly, all cases were valve prosthesis IE, of which six affected the aortic valve and six of seven patients were male. The time from valve insertion to the episode of IE varied from 4 weeks to 3 years. One patient died from the infection; one patient had a recurrent IE; and, in five cases, valve surgery was performed.

Table 3 Microbiology of true infections and contamination

\begin{tabular}{|c|c|c|c|}
\hline & True infection $(n=30)$ & Contamination $(n=309)$ & $p$ for difference \\
\hline Two or more positive blood cultures & $17(57)$ & $25(8.1)$ & $<0.0001$ \\
\hline Polymicrobial & $4(13)^{1}$ & $97(31)$ & 0.04 \\
\hline \multicolumn{4}{|l|}{ Species of Corynebacterium, $n(\%)$} \\
\hline Not determined to species & $4(13)$ & $81(25)$ & \multirow[t]{7}{*}{$<0.0001$} \\
\hline C. striatum & $8(27)$ & $34(11)$ & \\
\hline C. jeikeium & $4(10)$ & $3(1)$ & \\
\hline C. aurimucosum & $3(10)$ & $20(6.5)$ & \\
\hline C. amycolatum & $3(10)$ & $16(5.2)$ & \\
\hline C. afermentans & 0 & $46(15)$ & \\
\hline Other species & $8(27)^{2}$ & $109(37)^{3}$ & \\
\hline
\end{tabular}

${ }^{1}$ The other bacterium was a coagulase-negative Staphylococcus $(n=2)$, a Micrococcus $(n=1)$, and Cutibacterium acnes $(n=1) .{ }^{2}$ Other species were C. propinquum $(n=2)$, C. pseudodiphtericum $(n=1)$, C. glucoronolyticum $(n=1)$, C. mucifaciens $(n=1)$, C. riegelii $(n=1)$, C. stationis $(n=1)$, and C. ulcerans $(n=1) .{ }^{3}$ C. minutissimum $(n=15)$, C. imitans $(n=11)$, C. lipophiloflavum $(n=9)$, C. tuberculostearicum $(n=9), C$. coyleae $(n=8)$, C. propinquum ( $n=7), C$. mucifaciens $(n=7), C$. singulare $(n=7), C$. pseudodiphthericum $(n=6)$, C. glucoronolyticum $(n=6), C$. simulans $(n=4)$, C. riegelii $(n=4)$, C. stationis $(n=3), C$. macginleyi $(n=2)$, C. xerosis $(n=1), C$. falsenii $(n=1)$, C. resistens $(n=1)$, C. glaucum $(n=1)$, C. epidermidicanis $(n=1), C$. kroppenstedtii $(n=1), C$. durum $(n=1), C$. ureicelerivorans $(n=1)$, C. urealyticum $(n=1)$, C. ammoniagenes $(n=1)$, and C. pyruviciproducens $(n=1)$ 
Table 4 Characteristics of IE caused by Corynebacterium

\begin{tabular}{|c|c|c|c|c|c|c|c|c|c|}
\hline Gender & Age & Species & No. of blood cultures & Valve & TEE finding & Diagnosis & Treatment & Outcome & Comment \\
\hline Male & 66 & C. striatum & $3 / 3$ & $\mathrm{BAP}^{1}$ & Veg, abscess & Def & New BAP & Death & PCR on valve pos \\
\hline Female & 60 & C. jeikeium & $2 / 2$ & BAP & Abscess & Def & Homograft & Cured & PCR on valve pos \\
\hline Male & 56 & C. striatum & $2 / 2$ & BAP & Suspect & Poss & Conservative & Cured & \\
\hline Male & 75 & C. jeikeium & $1 / 3$ & BAP & Veg & Def & Homograft & Cured & $\begin{array}{l}\text { PCR on valve pos } \\
\text { CNS embolism }\end{array}$ \\
\hline Male & 70 & C. propinquum & $8 / 8$ & BAP & Normal & Poss & Conservative & Cured & \\
\hline Male & 69 & C. amycolatum & $2 / 4$ & BAP, BMP & MI & Def & Conservative & Relapsed & \\
\hline Male & 69 & C. amycolatum & $4 / 4$ & BAP, BMP & Veg & Def & New BMP & Cured & $\begin{array}{l}\text { PCR on valve pos } \\
\text { CNS embolism }\end{array}$ \\
\hline Male & 79 & C. pseudo-diphtheriticum & $2 / 3$ & BAP & Normal & Poss & Homograft & Cured & PET-CT pos \\
\hline
\end{tabular}

${ }^{1} B A P$, biological aorthic prosthesis; veg, vegetation; def, definite IE according to Duke; poss, possible according to Duke; BMP, biological mitral prosthesis

\section{Discussion}

This work demonstrates that Corynebacterium is a rare cause of severe infections and that bacteremia often represent contamination. Since our cohort is population-based, we conclude that the incidence of Corynebacterium IE is around one in a million per year, whereas true bacteremia occurs in around four cases per million inhabitants per year. On several occasions, very significant infections such as culture-proven Corynebacterium-caused IE or spondylodiscitis were diagnosed in patients with only one positive blood culture. This indicates that the definitions used for differentiating between true infection and contamination should take into account both bacteriological and clinical factors. Simple definitions such as one blood culture equals contamination will miss true infections. Our definition included a demand on clinical symptoms of infection, lack of other more likely causes of infection, and either two positive blood cultures or one positive blood culture in conjunction with an intravascular device or isolation of Corynebacterium at the site of a focal infection. This was adapted from previous works on coagulase-negative staphylococci [6] which, similar to Corynebacterium, often contaminate blood cultures and on rare occasions cause severe infections. Our definition is more sensitive than simple microbiological definitions based only on the number of positive cultures but there is also a risk for introducing false positives. Some of the episodes where Corynebacterium was isolated from two blood cultures and no focus was identified could certainly also represent contamination, whereas some likely represent missed cases of IE. We believe, however, that a more inclusive definition of true infection is important in order not to miss severe infections caused by Corynebacterium.

From our results, we conclude that the species of Corynebacterium is an important factor in determining if a finding is likely to represent true infection or contamination. Thus, the finding of $C$. jeikeium in blood is a strong suggestion of an underlying true infection whereas the isolation of C. afermentans strongly indicates contamination. We therefore suggest that clinical laboratories should determine the species of all corynebacterial isolated from blood so that clinicians can use this information to determine if a given isolate is a contaminant or relevant pathogen.

Corynebacterium IE has been described in numerous case reports but large case series or cohort-based studies are lacking. Our description of eight episodes of Corynebacteriumcaused IE is relevant as it represents a population-based account of this type of infection. Interestingly, all cases were related to valve prosthesis and this is clearly more than reported in a large review of published cases (19\%) [9]. The cases occurred both early after valve insertion and as late as 3 years after surgery suggesting that both intraoperative and hematogenous spread of bacteria occur. Our findings also demonstrate that the isolation of a Corynebacterium from blood in a patient with a heart valve prosthesis should evoke a strong suspicion of IE. Despite the potential severity of prosthesis-IE, the mortality was lower in our cohort (one of seven patients) than that reported from systematic reviews of case reports (40\%) [9]. This might be explained by a tendency of clinicians to mainly report dramatic presentations of IE. As in previous reviews on the topic, we observed a male dominance in Corynebacterium IE. Despite that our cohort is comparatively large, it is still too small to draw definite conclusions about the role of different Corynebacterium species in IE. It can be noted, however, that C. amycolatum, C. striatum, and C. jeikeium constitute a majority of cases and these species have been described previously to be common in Corynebacterium IE.

C. striatum has recently been shown to possess virulence factors, especially in terms of biofilm production, which implies that favorable conditions for the bacteria such as venous catheters constitute a risk environment for the growth of this particular bacterial species [17]. Indeed, spread and adaption of certain C. striatum clones in hospital settings has been 
demonstrated recently [18]. C. striatum is also known as a causative agent of a wide range of infections and for displaying or easily develop resistance to many antimicrobials [19-22]. Apart from IE and sepsis, C. striatum-related infections can arise from the respiratory tract, urinary tract, or wounds [23]. This is consistent with the findings in this study, where $C$. striatum was related to different types of infections.

The main strength of this study is that it is population-based and large. This allows for conclusions about incidences. Another strength is the combination of microbiological analyses and a careful analysis of clinical data which allow us to draw conclusions about the likelihood of different species to cause infection and contamination. The main limitation is that not all isolates were available for species determination and that there was a bias in which isolates had been saved. Isolates that the laboratory staff believed represented contaminations were less likely to be preserved, and this might have skewed the results from the species determination. The study was of course also limited by the retrospective design where only information recorded in the medical records were available for analysis. Despite that the study is population-based, of course, caution must be taken with extrapolation of the results to other geographical locations.

In conclusion, we demonstrate that Corynebacterium can cause severe infections and that species determination is an important tool to help clinicians in determining if a given patient has true infection caused by Corynebacterium. Therefore, we propose that microbiology laboratories should report the species of corynebacteria isolated from blood cultures.

Acknowledgments We acknowledge the invaluable support by Mrs. Lena Hyllebusk at the Department of Clinical Microbiology. This work was supported by the Swedish Government Fund for Clinical Research (ALF), the foundations of Österlund and Skåne University Hospital, and the Royal Physiographic Society in Lund. Part of this work was presented as an abstract at the Swedish infectious diseases meeting in Jönköping May 2019.

Funding Information Open access funding provided by Lund University.

\section{Compliance with ethical standards}

Conflict of interest The authors declare that they have no conflicts of interest.

Ethical approval All procedures performed were in accordance with the Swedish law on the ethics trial of research made on human subjects (SFS 2003:460) and were approved by the regional research ethics committee (ethics committee of Lund University, 2017/1002).

Open Access This article is distributed under the terms of the Creative Commons Attribution 4.0 International License (http:// creativecommons.org/licenses/by/4.0/), which permits unrestricted use, distribution, and reproduction in any medium, provided you give appropriate credit to the original author(s) and the source, provide a link to the Creative Commons license, and indicate if changes were made.

\section{References}

1. Bernard K (2012) The genus Corynebacterium and other medically relevant coryneform-like bacteria. J Clin Microbiol 50:3152-3158. https://doi.org/10.1128/JCM.00796-12

2. Kimura S-I, Gomyo A, Hayakawa J, Akahoshi Y, Harada N, Ugai T et al (2017) Clinical characteristics and predictive factors for mortality in coryneform bacteria bloodstream infection in hematological patients. J Infect Chemother 23:148-153. https://doi.org/10. 1016/j.jiac.2016.11.007

3. Ishiwada N, Watanabe M, Murata S, Takeuchi N, Taniguchi T, Igari $\mathrm{H}$ (2016) Clinical and bacteriological analyses of bacteremia due to Corynebacterium striatum. J Infect Chemother 22:790-793. https:// doi.org/10.1016/j.jiac.2016.08.009

4. Yanai M, Ogasawasa M, Hayashi Y, Suzuki K, Takahashi H, Satomura A (2018) Retrospective evaluation of the clinical characteristics associated with Corynebacterium species bacteremia. Braz J Infect Dis 22:24-29. https://doi.org/10.1016/j.bjid.2017.12.002

5. Leal SM, Jones M, Gilligan PH (2016) Clinical significance of commensal Gram-positive rods routinely isolated from patient samples. J Clin Microbiol 54:2928-2936. https://doi.org/10.1128/JCM. 01393-16

6. Finkelstein R, Fusman R, Oren I, Kassis I, Hashman N (2002) Clinical and epidemiologic significance of coagulase-negative staphylococci bacteremia in a tertiary care university Israeli hospital. Am J Infect Control 30:21-25

7. Rozdzinski E, Kern W, Schmeiser T, Kurrle E (1991) Corynebacterium jeikeium bacteremia at a tertiary care center. Infection 19:201-204

8. Brouqui P, Raoult D (2001) Endocarditis due to rare and fastidious bacteria. Clin Microbiol Rev 14:177-207. https://doi.org/10.1128/ CMR.14.1.177-207.2001

9. Belmares J, Detterline S, Pak JB, Parada JP (2007 6) Corynebacterium endocarditis species-specific risk factors and outcomes. BMC Infect Dis 7:4. https://doi.org/10.1186/1471-2334-7-4

10. Konrad R, Berger A, Huber I, Boschert V, Hörmansdorfer S, Busch U et al (2010) Matrix-assisted laser desorption/ionisation time-of-flight (MALDI-TOF) mass spectrometry as a tool for rapid diagnosis of potentially toxigenic Corynebacterium species in the laboratory management of diphtheria-associated bacteria. Euro Surveill 15

11. Alatoom AA, Cazanave CJ, Cunningham SA, Ihde SM, Patel R (2012) Identification of non-diphtheriae Corynebacterium by use of matrix-assisted laser desorption ionization-time of flight mass spectrometry. J Clin Microbiol 50:160-163. https://doi.org/10. 1128/JCM.05889-11

12. Mookadam F, Cikes M, Baddour LM, Tleyjeh IM, Mookadam M (2006) Corynebacterium jeikeium endocarditis: a systematic overview spanning four decades. Eur J Clin Microbiol Infect Dis 25: 349-353. https://doi.org/10.1007/s10096-006-0145-8

13. McElvania Tekippe E, Shuey S, Winkler DW, Butler MA, C-AD B (2013) Optimizing identification of clinically relevant grampositive organisms by use of the Bruker Biotyper matrix-assisted laser desorption ionization-time of flight mass spectrometry system. J Clin Microbiol 51:1421-1427. https://doi.org/10.1128/JCM. 02680-12

14. Charlson ME, Pompei P, Ales KL, MacKenzie CR (1987) A new method of classifying prognostic comorbidity in longitudinal studies: development and validation. J Chronic Dis 40:373-383

15. Li JS, Sexton DJ, Mick N, Nettles R, Fowler VG, Ryan T et al (2000) Proposed modifications to the Duke criteria for the diagnosis of infective endocarditis. Clin Infect Dis 30:633-638. https://doi. org/10.1086/313753

16. Berge A, Krantz A, Östlund H, Nauclér P, Rasmussen M (2018) The DENOVA score efficiently identifies patients with monomicrobial Enterococcus faecalis bacteremia where 
echocardiography is not necessary. Infection 30:633-636. https:// doi.org/10.1007/s15010-018-1208-3

17. de Souza C, Faria YV, de Sant'Anna LO, Viana VG, Seabra SH, de Souza MC et al (2015) Biofilm production by multiresistant Corynebacterium striatum associated with nosocomial outbreak. Mem Inst Oswaldo Cruz 110:242-248. https://doi.org/10.1590/ 0074-02760140373

18. Wang X, Zhou H, Chen D, Du P, Lan R, Qiu X et al Whole genome sequencing reveals a prolonged and persistent intra-hospital transmission of Corynebacterium striatum, an emerging multidrugresistant pathogen. J Clin Microbiol 2019:17. https://doi.org/10. 1128/JCM.00683-19

19. McElvania TeKippe E, Thomas BS, Ewald GA, Lawrence SJ, Burnham C-AD (2014) Rapid emergence of daptomycin resistance in clinical isolates of Corynebacterium striatum... a cautionary tale. Eur J Clin Microbiol Infect Dis 33:2199-2205. https://doi.org/10. 1007/s10096-014-2188-6

20. McMullen AR, Anderson N, Wallace MA, Shupe A, Burnham CA (2017) When good bugs go bad: epidemiology and antimicrobial resistance profiles of Corynebacterium striatum, an emerging multidrug-resistant, opportunistic pathogen. Antimicrob Agents Chemother 61:236. https://doi.org/10.1128/AAC.01111-17

21. Hahn WO, Werth BJ, Butler-Wu SM, Rakita RM (2016) Multidrug-resistant Corynebacterium striatum associated with increased use of parenteral antimicrobial drugs. Emerg Infect Dis 22: 1692. https://doi.org/10.3201/eid2211.160141

22. Werth BJ, Hahn WO, Butler-Wu SM, Rakita RM (2016) Emergence of high-level daptomycin resistance in Corynebacterium striatum in two patients with left ventricular assist device infections. Microb Drug Resist 22:233-237. https://doi. org $/ 10.1089 / \mathrm{mdr} .2015 .0208$

23. Lee PP, Ferguson DA, Sarubbi FA (2005) Corynebacterium striatum: an underappreciated community and nosocomial pathogen. J Inf Secur 50:338-343. https://doi.org/10.1016/j.jinf.2004.05. 005

Publisher's note Springer Nature remains neutral with regard to jurisdictional claims in published maps and institutional affiliations. 